\title{
Effects of sexual education mobile applications on men's sexual awareness and satisfaction: A randomized controlled trial
}

\author{
Mojtaba Gholizadeh 1, Hosein Shareh 2, Lida Jarahi 3, Mohammad Ahmadian \\ 4,Masoumeh Sarbaz 5, Mohammad Reza Darabi 6, Mohammad Reza Hasibian *7, Saeid \\ Eslami 8, 9 \\ 1: MSC Student in Department of Medical Informatics, Faculty of Medicine, Mashhad \\ University of Medical Sciences, Mashhad, Iran \\ 2: Assistant Professor of Clinical Psychology, Hakim Sabzevar University, Sabzevar, Iran. \\ 3: Department of Community Medicine, Faculty of Medicine, Mashhad University of \\ Medical Sciences, Mashhad, Iran \\ 4:MD., Mph, PhD Candidate in Traditional Iranian Medicine, School of Traditional Iranian \\ Medicine Mashhad University of Medical Sciences, Mashhad Iran \\ 5: Department of Medical Record and Health Information Technology, School of \\ Paramedical Sciences, Mashhad University of Medical Sciences, Mashhad, Iran \\ 6: Department of Urology, Facility of Medicine, Mashhad University of Medical Science, \\ Mashhad, Iran \\ 7: Department of Medical Informatics, Faculty of Medicine, Mashhad University of \\ Medical Sciences, Mashhad, Iran \\ 8: Pharmaceutical Research Center, Mashhad University of Medical Sciences, Mashhad, \\ Iran \\ 9: Department of Medical Informatics, Faculty of Medicine, Mashhad University of \\ Medical Sciences, Mashhad, Iran \\ *hasibianmr@mums.ac.ir
}

\begin{abstract}
BACKGROUND: Sexual education programs can improve sexual awareness and satisfaction. Yet, sex education is ignored in developing countries. Under such circumstances, we have used IT tools to improve sexual education.

OBJECTIVE: In this article, we used a mobile application (mHealth) to impart sex education.

Methods: A randomized controlled trial was held, in which participants were randomly assigned to one of two groups: The control group, with 25 participants, which received only counseling from sex therapists, and the intervention group, with 25 participants, which received the mobile application system in addition to counseling from sex therapists. Participants were persons referred to sex therapists at a clinic. In each group, sexual satisfaction and awareness were evaluated. We measured sexual satisfaction with the help of the Larson questionnaire and sexual awareness by the Ann Hooper questionnaire.

Results: Our data demonstrated that sexual satisfaction was not statistically significant $(\mathrm{P}=0.44)$, but awareness showed statistically significant differences $(\mathrm{P}=0.007)$ in the intervention vs. the control group. Also, the mean in both groups had statistically significant differences before and after the intervention $(\mathrm{P}=0.001)$.

Conclusion: Our results showed that mobile applications can improve sexual awareness but cannot affect sexual satisfaction in the short term.

Trial Registration: The clinical trial was registered with the Iranian Registry of Clinical Trials (IRCT) under registration ID: IRCT2016110130640N1

KEYWORDS: M-Health, mobile application, sexual education, sexual awareness, sexual satisfaction.
\end{abstract}


Medical Technologies Journal, Volume: 1, Issue: 4, October-December 2017, Pages:76-136. Doi : https://doi.org/10.26415/2572-004X-vol1iss4

\section{Declaration of conflicts}

This abstract is selected from the First International Congress of Diseases and Health Outcomes Registry and First National Congress of Medical Informatics, 14-17 February 2017, Mashhad, Iran

\section{Authors' biography}

No biography.

\section{References}

No references. 